\title{
Nonperturbative Many-Body Physics in the Open Bosonic String
}

\author{
V. Alan Kostelecký(1),(a) and Stuart Samuel ${ }^{(2),(b)}$ \\ (1) Physics Department, Indiana University, Bloomington, Indiana 47405 \\ ${ }^{(2)}$ Physics Department, The City College of New York, New York, New York 10031 \\ (Received 30 October 1989)
}

\begin{abstract}
We use covariant string field theory to explore many-body string physics. A candidate nonperturbative vacuum is identified via a level-truncation scheme. The breaking of internal and Lorentz symmetries is investigated. We show that tree-level string physics exhibits similarities to loop-level particle physics. The effective string coupling runs at tree level, inducing asymptotic freedom. This is a feature of any string theory. The running coupling has significant effects on the spectrum in the nonperturbative vacuum, including the disappearance of states.
\end{abstract}

PACS numbers: 11.17.+y

The remarkable phenomenological successes of particle field theories in recent years stem from many-body effects. These include asymptotic freedom and confinement in the strong interactions, and spontaneous symmetry breaking and the Higgs mechanism in the electroweak interactions. The phenomenological viability of superstring theories may also hinge on collective interactions. Certainly, superstrings in a perturbative firstquantized approach are poorly suited to describing the Universe: The dimensionality of spacetime is too large, and the spectrum is unrealistic.

This paper analyzes the string in its second-quantized version. We perform a semiclassical treatment of the covariant field theory of the open bosonic string. ${ }^{1-5}$ Our goal is insight, not phenomenology: Neither fermions nor gravity are present at this level.

In any field theory, vacuum structure is crucial. The open bosonic string is particularly interesting because tachyons destabilize the canonical 26-dimensional vacuum. Interactions may stabilize the theory in a different ground state, in which the tachyon field has a nonzero expectation value. ${ }^{6}$ Below, we identify a candidate vacuum, show that it is inaccessible in the zero-slope limit, and address the possibilities of spontaneous Lorentz- and internal-symmetry breaking.

We also demonstrate that the semiclassical string field theory has similarities to a non-Abelian particle gauge theory at the loop level. The string coupling runs at tree level, inducing tree-level asymptotic freedom. This substantially affects the physics in the nonperturbative vacuum. The number of states differs from the zero-slope count.

The basis of our analysis is a mass-level truncation scheme. Light fields often dominate physical processes. The reliability of this effect is further enhanced in covariant string field theory because the coupling between three states decreases exponentially with the sum of their squared masses, i.e., it behaves like $(4 / 3 \sqrt{3})^{n}$ $\approx \exp (-26 n)$, where $n=0,1,2, \ldots$ denotes the level number. The validity of the truncation scheme can be judged by direct comparison with available exact results and by the magnitude of higher-level corrections to lower-level results. Both methods indicate convergence of the scheme. ${ }^{7}$ In this paper, the fields incorporated are the tachyon and the massless vector. At this truncation level, one should see the qualitative features of higherlevel truncations while avoiding complexities.

The string field $\Psi$ is a linear combination of ordinary particle fields whose coefficients are the solutions of the first-quantized theory:

$$
\Psi^{a b}=\left(\phi^{a b}+i A_{\mu}^{a b} \alpha_{-1}^{\mu}-\alpha^{a b} b_{-1} c_{0}+\cdots\right)|0\rangle .
$$

Here, $\phi^{a b}$ is the tachyon field, $A_{\mu}^{a b}$ is the massless vector field, and $\alpha^{a b}$ is an auxiliary field. The superscripts are Paton-Chan factors, ${ }^{8}$ so that $\Psi$ and its particle-field components are square matrices. If the Paton-Chan group is $\mathrm{SO}(N)$ or $\mathrm{U}(N)$, odd mass-squared levels such as the tachyon form, respectively, symmetric or Hermitian matrices; even levels such as $A_{\mu}$ and $\alpha$ form antisymmetric or anti-Hermitian matrices. For U(1), our conventions agree with those of Ref. 7 if the $1 \times 1$ Hermitian and anti-Hermitian matrices are 1 and $-i$. The first-quantized string vacuum is $|0\rangle=c_{1}|\Omega\rangle$, where $|\Omega\rangle$ is the $\mathrm{SI}(2, R)$-invariant vacuum. ${ }^{9}$ The factor $i$ in $\mathrm{Eq}$. (1) ensures the reality condition ${ }^{1,10} \Psi^{b a \ddagger}=\Psi^{a b \dagger}$.

At this truncation level, the Langrangian is

$$
\begin{aligned}
\mathcal{L}=\operatorname{tr}\left[\frac{1}{2} \partial_{\mu} \phi \partial_{\mu} \phi-\frac{1}{2 \alpha^{\prime}} \phi \phi-\frac{1}{2} \partial_{\mu} A_{\nu} \partial^{\mu} A^{v}+\left(\frac{2}{\alpha^{\prime}}\right)^{1 / 2} \alpha \partial_{\mu} A^{\mu}-\frac{1}{\alpha^{\prime}} \alpha \alpha\right] \\
+q \operatorname{tr}\left\{\frac{1}{3}\left(\frac{2}{\alpha^{\prime}}\right)^{1 / 2} \gamma^{3} \tilde{\phi} \tilde{\phi} \tilde{\phi}+\gamma^{2} \partial^{\mu} \tilde{\phi}\left[\tilde{\phi}, \tilde{A} \tilde{A}_{\mu}\right]+\left(\frac{2}{\alpha^{\prime}}\right)^{1 / 2} \gamma \tilde{\phi}\left(\tilde{\alpha} \tilde{\alpha}-\tilde{A}_{\mu} \tilde{A}^{\mu}\right)+\left(\frac{\alpha^{\prime}}{2}\right)^{1 / 2} \gamma\left[\left(\partial_{\nu} \tilde{\phi}{\overleftrightarrow{\partial_{\mu}}}_{\mu} \tilde{A}^{v}\right) \tilde{A}^{\mu}-\left(\tilde{\phi} \vec{\partial}_{\mu} \tilde{A}^{v}\right) \partial_{v} \tilde{A}^{\mu}\right]\right. \\
\left.+\left[\tilde{A}^{\mu}, \tilde{A}^{v}\right] \partial_{\mu} \tilde{A}_{\nu}+\frac{\alpha^{\prime}}{6}\left\{3 \tilde{A}^{\mu}\left(\partial_{\lambda} \tilde{A}^{v}{\overleftrightarrow{\partial_{\mu}}}_{\partial_{v}} \tilde{A}^{\lambda}\right)+\left[\partial_{\lambda} \tilde{A}^{\mu}, \partial_{\mu} \tilde{A}^{v}\right] \partial_{v} \tilde{A}^{\lambda}\right\}-\tilde{A}^{\mu}\left(\tilde{\alpha}{\overleftrightarrow{\partial_{\mu}}}_{\mu} \tilde{\alpha}\right)\right\},
\end{aligned}
$$


where $\gamma=3 \sqrt{3} / 4, \alpha^{\prime}$ is the Regge slope, and $q$ is the Yang-Mills three-vector coupling. The latter is related to the on-shell three-tachyon coupling $g$ by $q$ $=g\left(2 \alpha^{\prime}\right)^{1 / 2} / 4$. Note that there is a suppression factor of $\gamma^{-n}$ in an interaction term, where $n$ is the sum of the mass-level numbers of the interacting fields. At all levels the particle fields $f$ in the interaction Lagrangian are smeared over a distance $\left(\alpha^{\prime}\right)^{1 / 2}$ :

$$
\tilde{f}=\exp \left[\alpha^{\prime} \ln (3 \sqrt{3} / 4) \partial_{\mu} \partial^{\mu}\right] f \text {. }
$$

On shell, $\tilde{\phi}=\gamma^{-1} \phi, \tilde{A}^{\mu}=A^{\mu}$, and $\tilde{\alpha}=\alpha$.

We seek a static vacuum solution. Setting $\tilde{f}=f$ and eliminating other momentum-dependent terms from the Lagrangian yields the following static potential:

$$
\begin{aligned}
V_{\text {static }}\left(\phi, A_{\mu}, \alpha\right)= & -\frac{1}{\alpha^{\prime}} \operatorname{tr}\left(\frac{1}{2} \phi \phi+\alpha \alpha\right) \\
& +\kappa g \operatorname{tr}\left(\phi \phi \phi \frac{2^{4}}{3^{2}} \phi\left(\alpha \alpha-A_{\mu} A^{\mu}\right)\right),
\end{aligned}
$$

where $\kappa=3 \sqrt{3} / 2^{7}$. An extremum of this potential represents a vacuum solution.

The usual perturbative solution about $\phi=A_{\mu}=\alpha=0$ is a local maximum. It is unstable and is an unsuitable choice of vacuum. A similar situation occurs in particle field theories: In the electroweak model, for example, the naive vacuum is perturbatively a local maximum in the scalar potential and the theory seeks a stable vacuum for which the scalars have expectation values.

To search for another solution, we take advantage of the global $\mathrm{SO}(N)$ or $\mathrm{U}(N)$ symmetry to diagonalize the matrix of the tachyon field. A local minimum in $V_{\text {static }}$ occurs at

$$
\begin{aligned}
& \left\langle\phi^{a b}\right\rangle:=\phi_{0} \delta^{a b}=\delta^{a b} / 3 \kappa g \alpha^{\prime} \approx 0.91 \delta^{a b} / g \alpha^{\prime}, \\
& \left\langle A_{\mu}^{a b}\right\rangle=0,\left\langle\alpha^{a b}\right\rangle=0 .
\end{aligned}
$$

Since $\langle\phi\rangle$ is of order $1 / g$, it is nonperturbative. ${ }^{6}$ The value of $V_{\text {static }}$ at the minimum is a contribution to the cosmological constant $\Lambda: \Lambda^{-1}=-2 \times 3^{3} \kappa^{2} g^{2} \alpha^{\prime 3} / N$.

When the next few levels are incorporated, the local minimum remains. ${ }^{7}$ The value of $\phi_{0}$ increases first by about $20 \%$ to $\phi_{0} \approx 1.083 / g \alpha^{\prime}$, and then to $\phi_{0} \approx 1.088 / g \alpha^{\prime}$. The magnitude of these changes suggests that the leveltruncation scheme yields qualitative features of a more exact treatment.

String field theory has a natural mechanism for the spontaneous breaking of the 26-dimensional Lorentz group ${ }^{11}$ due to the term $\tilde{\phi} \tilde{A}_{\mu} \tilde{A}^{\mu}$ in Eq. (2). A negative value of $\langle\phi\rangle$ would generate a negative squared mass for $A^{\mu}$. This in turn would induce an expectation value for $A_{\mu}$, breaking $\mathrm{O}(25,1)$ to $\mathrm{O}(24,1)$. However, our positive value of $\langle\phi\rangle$ excludes this possibility.

From Eqs. (2) and (3), we see that the string coupling runs already at tree level: The momentum-space couplings of three arbitrary states contains $\exp \left[-\alpha^{\prime}\left(p^{r}\right)^{2}\right.$ $x \ln (3 \sqrt{3} / 4)$, where $r=1,2,3$ is summed. It follows that the tree-level string field theory exhibits asymptotic freedom: The effective couplings decrease exponentially for large spacelike momenta with the scale being set by $\left(\alpha^{\prime}\right)^{1 / 2}$, which is of the order of the Planck length. ${ }^{12}$ Short-distance effects are perturbatively calculable in any vacuum state.

Tree-level asymptotic freedom should be a property of any string field theory. It is a consequence of the extended nature of the string, which causes good ultraviolet behavior by smearing interactions at short distances. Like loop effects in particle theories, the momentumdependent couplings renormalize propagator poles and the analysis of any scattering process requires wavefunction renormalization.

The running coupling causes difficulties with the determination of the spectrum of heavy states. This involves large squared timelike momenta $p_{0}^{2}$ for which the effective coupling is exponentially large: $g\left(p_{0}^{2}\right)=g$ $\times \exp \left[3 \alpha^{\prime} p_{0}^{2} \ln (3 \sqrt{3} / 4)\right]$. Strong-coupling effects render perturbation theory untrustworthy so that, once interactions are introduced, the location of poles in the $S$ matrix at momenta above $1 /\left(\alpha^{\prime}\right)^{1 / 2}$ is hard to establish. The high-mass spectrum in an interacting theory may be quite different from the free case.

A more subtle consequence of the running couplings is that some states may disappear entirely from the spectrum. Let us show how this arises in the level-one truncation scheme. The spectrum in the nonperturbative vacuum of Eq. (5) is analyzed by finding the zeros of the inverse propagators associated with the tachyon and vector fields. It simplifies matters to decompose fields into irreducible Lorentz multiplets. The field $A_{\mu}$ contains a scalar $A^{L}$ and a vector $A_{\mu}^{T}$, where we define $A_{\mu}$ $=A_{\mu}^{T}+\partial_{\mu} A^{L} /\left(\left|\partial^{2}\right|\right)^{1 / 2}$ with $A^{L}=\partial^{\lambda} A_{\lambda} /\left(\left|\partial^{2}\right|\right)^{1 / 2}$.

The analysis of small oscillations in the nonperturbative vacuum reveals no mixing between the scalars $\phi$ and $A^{L}$. In Euclidean momentum space, the quadratic form $Q_{\phi}\left(p^{2}\right)$ associated with $\phi$ is

$$
Q_{\phi}\left(p^{2}\right)=p^{2}-\frac{1}{\alpha^{\prime}}+\frac{2}{\alpha^{\prime}} \exp \left[-2 \alpha^{\prime} p^{2} \ln \left(\frac{3 \sqrt{3}}{4}\right)\right] \text {. }
$$

A propagator pole at $p^{2}=-p_{0}^{2}$ exists if $Q_{\phi}\left(-p_{0}^{2}\right)=0$. However, $Q_{\phi}\left(-p_{0}^{2}\right)$ is positive for all values of $p_{0}^{2}$.

The state associated with $\phi$ has disappeared from the spectrum. This effect can be attributed to the running coupling and to the presence of the exponentials of $p^{2}$ in the propagator. Without this transcendental behavior, i.e., if $\phi$ instead of $\tilde{\phi}$ appeared in Eq. (2), there would be a state associated with the field $\phi$ with a mass of $1 /\left(\alpha^{\prime}\right)^{1 / 2}$.

Usually, in particle field theories the total number of degrees of freedom is unaffected by the presence of nonzero expectation values. In the electroweak model, for example, the number of degrees of freedom is preserved when the three intermediate vector bosons ac- 
quire masses by absorbing three scalar states. The equation $Q_{\phi}\left(-p_{0}^{2}\right)=0$ avoids this constraint by virtue of its transcendental nature. ${ }^{13}$ The string condensate in the nonperturbative ground state prevents the propagation of $\phi$ beyond the Planck length.

Next we consider $A_{\mu}$. The quadratic forms for $A_{\mu}^{T}$ and $A^{L}$ are, respectively,

$Q_{A_{\mu}^{T}}\left(p^{2}\right)=p^{2}+\frac{2^{5}}{3^{3} \alpha^{\prime}} \exp \left[-2 \alpha^{\prime} p^{2} \ln \left(\frac{3 \sqrt{3}}{4}\right)\right]$

and

$Q_{A^{L}}\left(p^{2}\right)=p^{2}+\left(\frac{2^{5}}{3^{3} \alpha^{\prime}}+\frac{2^{4} p^{2}}{3^{3}}\right) \exp \left[-2 \alpha^{\prime} p^{2} \ln \left(\frac{3 \sqrt{3}}{4}\right)\right]$

We find that $Q_{A_{\mu}^{T}}\left(p^{2}=-m^{2}\right)$ never vanishes as a function of $m^{2}$, so no real pole appears for the transverse vec- tor field. In contrast, the scalar $A^{L}$ has a real pole at $m_{A^{L}}=1 /\left(\alpha^{\prime}\right)^{1 / 2}$. If the exponential factors were absent in Eqs. (7) and (8), there would be real poles for $A_{\mu}^{T}$ at $-2^{5} / 3^{3} \alpha^{\prime} \approx-1.19 / \alpha^{\prime}$ and for $A^{L}$ at $p^{2}=-2^{5} / 43 \alpha^{\prime}$ $\approx-0.74 / \alpha^{\prime}$.

Loop effects probably produce further vacuum instability due to the appearance of the closed string and its tachyon. The nature of the ground state must again be determined. If the spectrum of the full theory resembles that found above, i.e., if it contains neither tachyons nor massless states, then infrared problems are absent and the bosonic string theory in the true vacuum is finite. A complete analysis of nonperturbative finiteness awaits the formulation of a consistent closed-string field theory.

Let us next address the breaking of internal symmetries. String field theory is a gauge theory with an infinite number of gauge transformations that form a string field $\Lambda^{a b}$. The lowest-level particle field $\lambda^{a b}$ in $\Lambda^{a b}=\left(-\lambda^{a b}+\cdots\right)|\Omega\rangle$ generates the following transformation at our truncation order:

$$
\begin{aligned}
\delta \phi= & q \gamma^{2}[\tilde{\lambda}, \tilde{\phi}]+q \gamma\{\tilde{\lambda}, \tilde{\alpha}\}-q \gamma\left(\frac{\alpha^{\prime}}{2}\right)^{1 / 2}\left(2\left\{\partial_{\mu} \tilde{\lambda}, \tilde{A}^{\mu}\right\}+\left\{\tilde{\lambda}, \partial_{\mu} \tilde{A}^{\mu}\right\}\right), \\
\delta A_{\mu}= & \partial_{\mu} \lambda+q\left[\tilde{\lambda}, \tilde{A}_{\mu}\right]+q\left(\frac{\alpha^{\prime}}{2}\right)^{1 / 2}\left(\left[\tilde{\alpha}, \partial_{\mu} \tilde{\lambda}\right]+\left[\tilde{\lambda}, \partial_{\mu} \tilde{\alpha}\right]\right)+q \gamma\left(\frac{\alpha^{\prime}}{2}\right)^{1 / 2}\left(\left\{\tilde{\lambda}, \partial_{\mu} \tilde{\phi}\right\}-\left\{\partial_{\mu} \tilde{\lambda}, \tilde{\phi}\right\}\right) \\
& +q \frac{\alpha^{\prime}}{2}\left(\left[\partial_{\mu} \tilde{\lambda}, \partial^{v} \tilde{A}_{v}\right]-\left[\tilde{\lambda}, \partial_{\mu} \partial^{v} \tilde{A}_{v}\right]+2\left[\partial^{v} \partial_{\mu} \tilde{\lambda}, \tilde{A}_{v}\right]-2\left[\partial^{v} \tilde{\lambda}_{,} \partial_{\mu} \tilde{A}_{v}\right]\right), \\
\delta \alpha= & \left(\frac{\alpha^{\prime}}{2}\right)^{1 / 2} \partial^{\mu} \partial_{\mu} \lambda+\frac{q}{2}[\tilde{\lambda}, \tilde{\alpha}] \frac{q}{2}\left(\frac{\alpha^{\prime}}{2}\right)^{1 / 2}\left(\left[\tilde{\lambda}, \partial^{\mu} \tilde{A}_{\mu}\right]+2\left[\partial^{\mu} \tilde{\lambda}, \tilde{A}_{\mu}\right]\right)-\frac{q}{2} \gamma\{\tilde{\lambda}, \tilde{\phi}\},
\end{aligned}
$$

where a tilde over a commutator or anticommutator indicates smearing the product of the two fields using Eq. (3). In the zero-slope limit, the field transforms as a non-Abelian $\mathrm{SO}(N)$ or $\mathrm{U}(N)$ particle gauge theory. Explicit calculation reveals that Eq. (2) is invariant under Eq. (9) at orders $g^{0}$ and $g^{1}$ but not at order $g^{2}$. Order$g^{2}$ invariance is recovered only when all levels are incorporated.

From Eq. (9), no spontaneous $\lambda$-symmetry breaking occurs at this truncation order because $\langle\phi\rangle$ is proportional to the identity matrix and commutes with $\lambda$. To establish which string symmetries are spontaneously broken in the full theory, we separate each of the fields $\Psi$ and $\Lambda$ into two groups: $O_{\Psi}$ and $E_{\Psi}$ for $\Psi, O_{\Lambda}$ and $E_{\Lambda}$ for $\Lambda$. Here, $O$ and $E$ represent odd and even mass-squared levels, respectively. As matrices, $O^{\dagger}=O$ and $E^{\dagger}=-E$. The field $\phi$ is in $O_{\Psi}$ and $\lambda$ is in $E_{\Lambda}$. When higher levels are incorporated, other scalars acquire expectation values but all of them are in $O_{\Psi} \cdot{ }^{7}$ Hence, $\left\langle E_{\psi}\right\rangle=0$ but $\left\langle\operatorname{tr}\left(O_{\Psi}\right)\right\rangle \neq 0$. Unbroken symmetries leave $\left\langle\operatorname{tr}\left(O_{\Psi}\right)\right\rangle$ invariant; broken symmetries transform $\left\langle\operatorname{tr}\left(O_{\Psi}\right)\right\rangle$. Since the structure of the string gauge transformations is

$\delta O_{\Psi}=Q O_{\Lambda}+\left[E_{\Lambda}, O_{\Psi}\right]+\left\{E_{\Lambda}, E_{\Psi}\right\}+\left\{O_{\Lambda}, O_{\Psi}\right\}+\left[O_{\Lambda}, E_{\Psi}\right]$,

the low-energy non-Abelian $\lambda$ symmetry is unbroken to all truncation orders. The broken symmetries correspond to the trace of the odd levels of $\Lambda$.

Usually, in particle theories there is a massless vector boson for each unbroken gauge symmetry. String theory is different. In the free theory, there are an infinite number of gauge symmetries but only one massless vector field $A_{\mu}^{a b}$. Furthermore, as seen above, the absence of spontaneous gauge-symmetry breaking does not guarantee the masslessness of the vector gauge bosons.

It is interesting to compare these unusual effects with features of the strong interactions. In quantum chromodynamics, the SU(3) color group is unbroken but the theory contains no massless vector bosons. The gluons, like $A_{\mu}^{a b}$ above, do not manifest themselves as poles in the $S$ matrix. These effects are probably a consequence of color infrared slavery, a property absent in tree-level string physics because the effective couplings are finite at zero momentum. In contrast, the string effects are due to asymptotic freedom.

The above discussion implicitly assumes that the nonperturbative vacuum is perturbatively stable at the quantum-mechanical level. This is actually the case. The relevant criterion is that the Euclidean-space propagators of the small field fluctuations never diverge, i.e., 
that the quadratic forms never vanish. ${ }^{7}$ We have confirmed numerically that Eqs. (6)-(8) are nonvanishing in Euclidean space for all $p^{2}$. The stability criterion is violated by the canonical vacuum, where the tachyon quadratic form vanishes at $p^{2}=1 / \alpha^{\prime}$.

Nonperturbative quantum-mechanical stability is harder to address. The local minimum of the potential is not a global minimum and may be subject to barrier penetration. Since there is a tunneling-suppression factor for each degree of freedom and since string theory involves an infinite number of particle fields, string tunneling should be inhibited relative to particle tunneling. This question deserves further attention.

Unless all nonzero expectation values are very small compared to $1 / g \alpha^{\prime}$, the spectrum of any string theory in a nonperturbative vacuum is probably significantly changed relative to the free case because the powers of $\exp \left(\alpha^{\prime} p^{2}\right)$ that appear in quadratic forms are likely to induce mass shifts of Planck-scale order. Furthermore, barring the vanishing of key couplings the gauge symmetries do not necessarily maintain masslessness of vector bosons, as we have seen. This brings into question whether a nonperturbative vacuum can be phenomenologically viable. However, since the standard model and grand unified theories require the breaking of symmetries, nonperturbative many-body physics at some scale is necessary. These contrasting facts emphasize the long-standing question concerning the construction of a realistic string model: How to generate a light spectrum for the observed particles when the natural scale is the Planck mass.

This work was supported in part by the United States Department of Energy under Contracts No. DE-AC02-
84ER40125 and No. DE-AC02-83ER40107.

(a) BITNET address: KOSTELEC @ IUBACS.

(b) BITNET address: SAMUEL @ CCNYSCI.

${ }^{1}$ E. Witten, Nucl. Phys. B268, 253 (1986).

${ }^{2}$ D. Gross and A. Jevicki, Nucl. Phys. B283, 1 (1987); B287, 225 (1987).

${ }^{3}$ E. Cremmer, A. Schwimmer, and C. Thorn, Phys. Lett. B 179, 57 (1986).

${ }^{4}$ S. Samuel, Phys. Lett. B 181, 255 (1986).

${ }^{5}$ N. Ohta, Phys. Rev. D 34, 3785 (1986).

${ }^{6}$ Any nonzero expectation values are necessarily nonperturbative in the string coupling and Regge slope. See V. A. Kostelecký and S. Samuel, Phys. Lett. B 207, 169 (1988).

${ }^{7}$ V. A. Kostelecký and S. Samuel, Nucl. Phys. B (to be published)

${ }^{8}$ J. E. Paton and Chan H.-M., Nucl. Phys. B10, 516 (1969).

${ }^{9}$ D. Friedan, E. Martinec, and S. Shenker, Nucl. Phys. B271, 93 (1986).

${ }^{10}$ S. Samuel, Nucl. Phys. B296, 187 (1988).

IIV. A. Kostelecký and S. Samuel, Phys. Rev. D 39, 683 (1989); Phys. Rev. Lett. 63, 224 (1989); Phys. Rev. D 40, 1886 (1989).

${ }^{12}$ This phenomena occurs in an off-shell region of momentum space. The high-energy behavior of on-shell processes has been analyzed in D. J. Gross and P. F. Mende, Nucl. Phys. B303, 407 (1988).

${ }^{13}$ There are many complex solutions. The most trustworthy have smallest $\left|p_{0}\right|$ and occur at $p^{2} \approx(0.24 \pm 1.9 i) / \alpha^{\prime}$. If such complex poles appear in a physical $S$-matrix element, they are likely to represent minuscule enhancements of the background in scattering or tiny violations of casuality. The latter might be expected in string theory since strings are nonlocal objects on the scale $\left(\alpha^{\prime}\right)^{1 / 2}$. 